\title{
Multiresolution Subdivision Snakes
}

\author{
Anaïs Badoual, Daniel Schmitter, Virginie Uhlmann, and Michael Unser
}

\begin{abstract}
We present a new family of snakes that satisfy the property of multiresolution by exploiting subdivision schemes. We show in a generic way how to construct such snakes based on an admissible subdivision mask. We derive the necessary energy formulations and provide the formulas for their efficient computation. Depending on the choice of the mask, such models have the ability to reproduce trigonometric or polynomial curves. They can also be designed to be interpolating, a property that is useful in user-interactive applications. We provide explicit examples of subdivision snakes and illustrate their use for the segmentation of bioimages. We show that they are robust in the presence of noise and provide a multiresolution algorithm to enlarge their basin of attraction, which decreases their dependence on initialization compared to singleresolution snakes. We show the advantages of the proposed model in terms of computation and segmentation of structures with different sizes.
\end{abstract}

Index Terms-Multiresolution, subdivision, snake, minimumsupport, Deslauriers-Dubuc, segmentation, interpolation.

\section{INTRODUCTION}

A CTIVE contours, also called "snakes", are popular models for the segmentation of biomedical images [1]-[6]. They consist in an initial shape that evolves towards the boundary of the object of interest. The evolution is guided by the choice of an appropriate energy term to be minimized. Different snake models have been proposed [7], [8]. They can be categorized by the way their shape is described: either discretely or in the continuous domain. In particular, there are point-snakes and parametric snakes. Point-snakes have a simple discrete representation. The shape is described by a set of ordered points [9]. However, they rely on a large number of parameters (i.e., the snake points), which requires an internal regularization to enforce smooth boundaries and makes the optimization more challenging. Parametric snakes have a continuous representation by using basis functions. They require fewer parameters (i.e., control points), which results in a faster optimization and better robustness. They are usually built in a way that ensures continuity and smoothness. However, the shape that the snake can reproduce is limited by its parametrization. We propose in this paper a geometric representation that combines the advantages of point-snakes and parametric snakes. In our representation, the curve is driven by a discrete set of a few master points, called control points, that

Manuscript received April 27, 2016; revised September 28, 2016 and December 1, 2016; accepted December 13, 2016. Date of publication December 21, 2016; date of current version January 20, 2017. This work was supported by the Swiss National Science Foundation under Grant 200020162343. The Associate Editor coordinating the review of this manuscript and approving it for publication was Prof. Gustavo Kunde Rohde.

The authors are with the Biomedical Imaging Group, École Polytechnique Fédérale de Lausanne, 1015 Lausanne, Switzerland.

Color versions of one or more of the figures in this paper are available online at http://ieeexplore.ieee.org.

Digital Object Identifier 10.1109/TIP.2016.2644263 are the parameters of the model. Then, slave points describing the curve are generated by specific iterative procedures. The property that makes it possible is called subdivision [10]-[13]. It is tightly linked to the theory of wavelets [14] and allows one to describe a contour or a surface by an initial discrete and finite set of control points which, by the iterative application of refinement rules, becomes continuous in the limit. The discrete nature of the representation is convenient in practical applications. At the same time, it implicitly yields a continuously defined model whose smoothness depends on the particular choice of the subdivision mask. The main benefits of subdivision schemes are their simplicity of implementation, the possibility to control their order of approximation, and their multiresolution property, which allows for the contour of a shape to be represented at varying resolutions.

The use of subdivisions for the construction of segmentation models was pioneered by [15] and [16] for Doo-Sabin surfaces [17] and the DLG-scheme [18], respectively. In the first case, left ventricles are modeled whereas, in the second case, they improved editing semantics of traditional snakes. In this paper, we propose a general approach that remains valid for any subdivision scheme as we derive the construction of a $2 \mathrm{D}$ subdivision snake in a generic way. The primary contributions of this work are: 1) a new geometrical representation based on subdivision. A crucial aspect is the choice of the subdivision mask that determines important properties of the model such as its approximation properties, the capability of reproducing circular, elliptical, or polynomial shapes [19], as well as the possibility of being interpolatory [20], [21] or not; 2 ) the derivation of associated energy functions such as regionand edge-based terms; 3) the presentation of an integrated strategy where the snake is optimized in a coarse-to-fine fashion. This multiscale approach is algorithmic and inherently recursive: We increase the number of points describing the curve as the algorithm progresses to the solution; at each step, the scale of the image feature (on which the optimization is performed) is matched to the density of the point cloud. This speeds up the computation and increases the robustness.

We give several examples of explicit constructions of subdivision snakes. We illustrate their use on real images as well as on test data simulating real biological conditions. We compare our proposed model to existing parametric snakes and measure its robustness and accuracy w.r.t. noise and initialization. Specifically, we show that the proposed coarseto-fine approach allows the optimizer to 1) have a larger basin of attraction which makes it robust to initial conditions; 2) escape some local optima; 3) be efficient by progressively increasing the snake resolution; 4) delineate structures of different sizes contained within an image without having to adapt the initialization. 


\section{A. Organization of the Article}

In Section II, we introduce and describe the theory of subdivision that is relevant to the construction of curves. In Section III, we fully specify the construction of generic subdivision snakes. We also describe the proposed multiresolution algorithm for the optimization. In Section IV, we present several types of multiresolution snakes where the subdivision masks possess various properties such as being interpolatory, having different sizes of support, and reproducing polynomials. In Section V, we show how subdivision schemes can be used to reproduce trigonometric functions for the construction of elliptic and circular curves. In Section VI, we perform an extensive validation of subdivision snakes based on test data where the ground truth is known as well as on real biological data. Finally, in Section VII, we discuss the choice of the subdivision mask according to the application and we provide a method to choose the parameters of the multiresolution algorithm.

\section{Closed Subdivision Curves}

\section{A. Notations}

We represent by $\mathbf{p}[\cdot]$ a discrete sequence of points $\mathbf{p}[m]=$ $\left(p_{1}[m], p_{2}[m]\right)$, indexed by $m \in \mathbb{Z}$, where $p_{1}$ and $p_{2}$ are the corresponding coordinates. We write $\mathbf{p}_{(k)}[\cdot]=$ $\left(p_{1(k)}[\cdot], p_{2(k)}[\cdot]\right)$ to describe a $\left(2^{k} N_{0}\right)$-periodic sequence, $k \geq 0$, with the property that $\mathbf{p}_{(k)}\left[m+n 2^{k} N_{0}\right]=\mathbf{p}_{(k)}[m]$, $\forall n \in \mathbb{Z}$. The discrete convolution of $\mathbf{p}_{(k)}[\cdot]$ with a scalar mask $h[\cdot]$ is defined as

$$
\left(h * \mathbf{p}_{(k)}\right)[m]=\sum_{n=-\infty}^{+\infty} h[m-n] \mathbf{p}_{(k)}[n] .
$$

\section{B. Subdivision Schemes}

A subdivision scheme generates a continuously defined function as the limit of an iterative algorithm that is applied to an initial set of $N_{0}$ control points. A refinement rule is applied repeatedly $k$ times to double the number of points at each iteration, ultimately yielding a set of $2^{k} N_{0}$ points. Note that, at each iteration, the new set of points does not necessary contain the previous ones. The subdivision scheme is said to be convergent when the set of points converges to the continuous curve $\mathbf{r}=\left(r_{1}, r_{2}\right)$ with $r_{1}, r_{2} \in \mathcal{C}^{0}$ as $k \rightarrow \infty$.

A closed curve at resolution $k$ is represented by a $\left(2{ }^{k} N_{0}\right)$ periodized coordinate sequence $\mathbf{p}_{(k)}[\cdot]$. The refinement rule from $(k-1)$ to $k$ is defined by

$$
\mathbf{p}_{(k)}[m]=h * \mathbf{p}_{(k-1)_{\uparrow}}[m],
$$

where $h$ is the subdivision mask of the subdivision scheme [22] and $\uparrow_{2}$ denotes an upsampling by a factor of 2 , given by

$$
\mathbf{p}_{(k)_{\uparrow}}[m]= \begin{cases}\mathbf{p}_{(k)}[n], & m=2 n \\ 0, & \text { otherwise. }\end{cases}
$$

In practice, the mask $h$ has a finite number of non-zero elements so that the infinite sum in (1) is often reduced to a finite one. Applying (1) iteratively, we can express the refinement rule as a function of the initial set of control

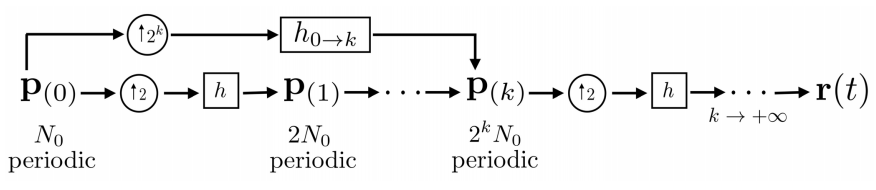

Fig. 1. Flowchart of a subdivision scheme. The periodic sequence $\mathbf{p}_{(k)}$, associated to the subdivision points at iteration $k$, converges to the continuous curve $\mathbf{r} ; h$ is the subdivision mask and the sequence $h_{0 \rightarrow k}$, defined by (3), allows one to obtain $\mathbf{p}_{(k)}$ directly from the initial set of control points $\mathbf{p}_{(0)}$.

points $\mathbf{p}_{(0)}$. The subdivision points at the $k$ th iteration $(k \geq 1)$ are thereby described by

$$
\mathbf{p}_{(k)}=h_{0 \rightarrow k} * \mathbf{p}_{(0)_{\uparrow_{2}}},
$$

where

$$
h_{0 \rightarrow k}=h_{\uparrow_{2^{k-1}}} * h_{\uparrow_{2^{k-2}}} * \cdots * h_{\uparrow_{2}} * h .
$$

The derivation of (2) is given in Appendix A. Note that each set of points $\mathbf{p}_{(k)}$ is encoded with the $N_{0}$ control points $\left\{\mathbf{p}_{(0)}[m]\right\}_{m \in\left[0 \ldots N_{0}-1\right]}$. The subdivision scheme is illustrated in Figures 1 and 2.

In the following, the term control points designates the $N_{0}$ initial points $\left\{\mathbf{p}_{(0)}[m]\right\}_{m \in\left[0 \ldots N_{0}-1\right]}$ and the term subdivision points describes the $2^{k} N_{0}$ points $\left\{\mathbf{p}_{(k)}[m]\right\}_{m \in\left[0 \ldots 2^{k} N_{0}-1\right]}$ at the $k$ th iteration $(k \geq 1)$.

\section{Convergent Subdivision Schemes}

Let $h$ be a subdivision mask with $z$-transform ${ }^{1} H(z)=$ $\sum_{n \in \mathbb{Z}} h[n] z^{n}$. A necessary condition for the corresponding subdivision scheme to be convergent is that $\sum_{n \in \mathbb{Z}} h[2 n]=$ $\sum_{n \in \mathbb{Z}} h[2 n+1]=1$ [23]. The subdivision scheme thus reproduces constants and $H(z)=(1+z) B(z)$, where $B(z)$ is a Laurent polynomial and $B(1)=1$ [24].

For any convergent subdivision scheme, the points of the sequence $\mathbf{p}_{(k)}$, as $k \rightarrow \infty$, sample the limit curve $\mathbf{r}$, in the sense that [24]-[26]

$$
\left.\mathbf{r}(t)\right|_{t=\frac{m}{2^{k}}}=\mathbf{p}_{(k)}[m] .
$$

When the coordinates function of the curve satisfy $r_{1}, r_{2} \in \mathcal{C}^{1}$, the derivative $\dot{\mathbf{r}}=\frac{\mathrm{d} \mathbf{r}}{\mathrm{d} t}$ is also sampled by

$$
\left.\dot{\mathbf{r}}(t)\right|_{t=\frac{m}{2^{k}}}=2^{k}\left(\mathbf{p}_{(k)}[m+1]-\mathbf{p}_{(k)}[m]\right)
$$

in the limit case $k \rightarrow \infty$ [25], [27]. The derivation of (5) is given in Appendix B. A necessary and sufficient condition for a subdivision scheme to converge uniformly to a continuous limit function is [23], [27]

$$
\left\{\begin{array}{l}
H(1)=2 \\
H(-1)=0 \\
\max _{m}\left|h_{0 \rightarrow k}[m+1]-h_{0 \rightarrow k-1}[m]\right| \underset{k \rightarrow+\infty}{\longrightarrow} 0 .
\end{array}\right.
$$

In practice, six iterations are enough to have satisfactory convergence (see Figure 2).

\footnotetext{
${ }^{1}$ This is the conventional definition of the $z$-transform used in subdivision theory.
} 


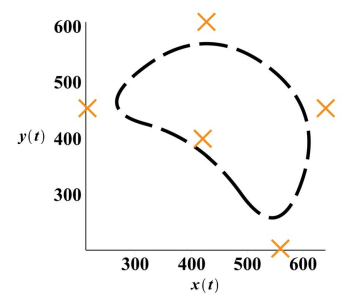

(a) $\mathbf{p}_{(0)}: N_{0}$-periodic

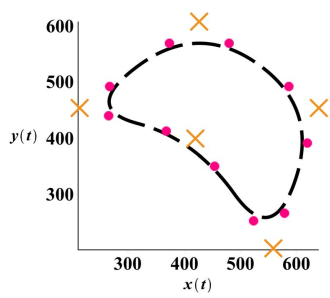

(b) $\mathbf{p}_{(1)}: 2 N_{0}$-periodic

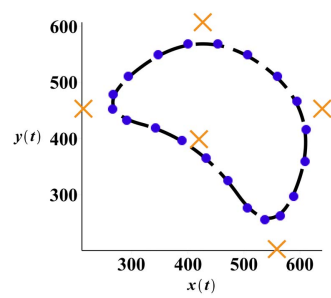

(c) $\mathbf{p}_{(2)}: 2^{2} N_{0}$-periodic

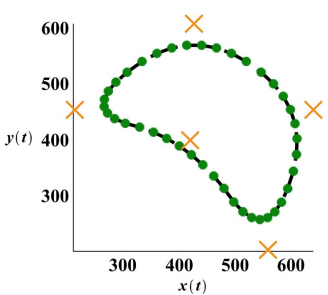

(d) $\mathbf{p}_{(3)}: 2^{3} N_{0}$-periodic

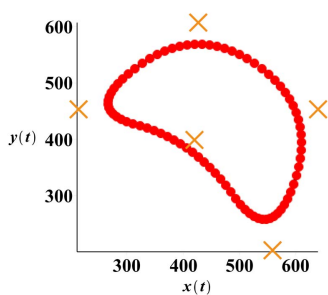

(e) $\mathbf{p}_{(4)}: 2^{4} N_{0}$-periodic

Fig. 2. Illustration of a non-interpolating subdivision scheme. (a) Control points. Dots in (b)-(e): Subdivision points of the first four iterations. As the points become denser with each iteration, they converge to the continuous curve $\mathbf{r}$ (dashed black line), which is still encoded by the five control points (orange crosses).

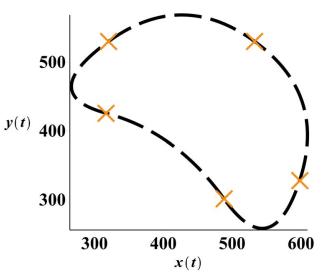

(a) $\mathbf{p}_{(0)}: N_{0}$-periodic

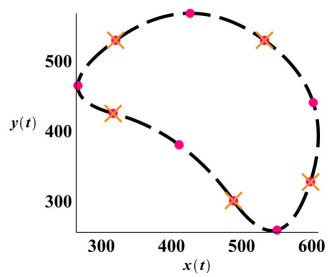

(b) $\mathbf{p}_{(1)}: 2 N_{0}$-periodic

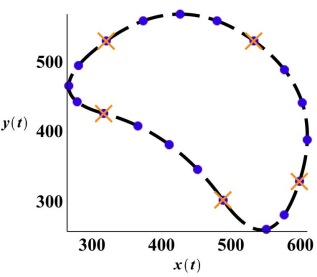

(c) $\mathbf{p}_{(2)}: 2^{2} N_{0}$-periodic

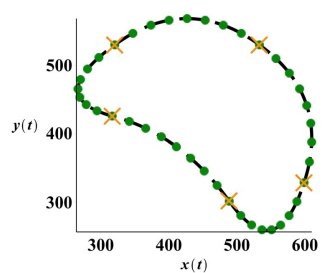

(d) $\mathbf{p}_{(3)}: 2^{3} N_{0}$-periodic

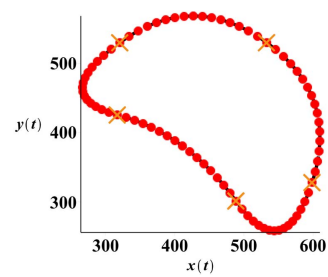

(e) $\mathbf{p}_{(4)}: 2^{4} N_{0}$-periodic

Fig. 3. Illustration of an interpolating subdivision scheme. We show the control points (a) and the first four sets of subdivision points (dots in (b)-(e)). They interpolate the limit curve $\mathbf{r}$ (dashed black line), which is still encoded by the five control points (orange crosses).

\section{Interpolating Subdivision Schemes}

A subdivision scheme is said to be interpolating if $h[2 m]=\delta[m]$, where $\delta$ denotes the Kronecker delta. It means that, at each step $k$, the subdivision points interpolate the limit

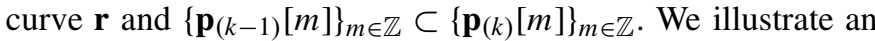
interpolating subdivision scheme in Figure 3.

\section{SUBDIVISION SNAKES}

Snakes are active-contour models that are optimized through the minimization of an energy term. The snake is iteratively updated until the minimum of the energy functional is obtained. In this section, we explain the construction of subdivision snakes and propose an integrated multiresolution optimizer.

\section{A. Geometrical Representation of the Snake}

In order to construct a snake, a suitable model to represent shapes needs to be established. Geometric requirements need to be taken into account such as shape-reproduction properties or smoothness constraints. The geometric reproduction properties of a model determine which configurations the snake can adopt, such as polynomial or elliptic. We implicitly describe the contour of the snake by the continuous limit curve $\mathbf{r}$ of the convergent subdivision scheme. This implies that the properties of the snake are determined by the choice of the mask $h$. An important requirement for the construction of the snake is that the representation model (4) be affine invariant. This property insures that a curve is described independently from its location and orientation.

Definition 1: A subdivision scheme is said to be affine invariant if, for any $(2 \times 2)$ matrix $\mathbf{A}$ and translation vector $\mathbf{b} \in \mathbb{R}^{2}$, the following relation holds:

$$
\begin{aligned}
\lim _{k \rightarrow \infty} h_{0 \rightarrow k} *\left(\mathbf{A p}_{(0)}+\mathbf{b}\right)_{\uparrow_{2^{k}}} & =\mathbf{A}\left(\lim _{k \rightarrow \infty} h_{0 \rightarrow k} * \mathbf{p}_{(0)}\right)+\mathbf{b} \\
& =\mathbf{A r}+\mathbf{b} .
\end{aligned}
$$

Proposition 1: Every convergent subdivision scheme is affine invariant.

The derivation of Proposition 1 is given in Appendix C.

\section{B. Snake Energies and Optimization}

Another important aspect in the construction of a snake is the formulation of a suitable energy functional. The choice of this energy term is crucial because it determines the quality of the outcome. We use an image energy, which is purely data driven. It involves a convex combination of an edge-based term using gradient information to detect contours [1], [28], [29] and a region-based term which uses statistical information to distinguish different homogeneous regions [30], [31]. We express the total snake energy as

$$
E_{\text {snake }}\left(f, \mathbf{p}_{(k)}\right)=b E_{\text {edge }}\left(f, \mathbf{p}_{(k)}\right)+(1-b) E_{\text {region }}\left(f, \mathbf{p}_{(k)}\right),
$$

where $b \in[0,1]$, is a tradeoff parameter that balances the contribution of the two energies, $f$ is the image to be segmented, and $\mathbf{p}_{(k)}$ describes the contour of the snake. The optimization is computed as a function of the control points $\mathbf{p}_{(0)}$ and the optimum is obtained as

$$
\mathbf{p}_{(0)_{\mathrm{opt}}}=\underset{\mathbf{p}_{(0)}}{\arg \min } E_{\text {snake }}\left(f, \mathbf{p}_{(k)}\right) .
$$

We propose

$$
E_{\text {edge }}\left(f, \mathbf{p}_{(k)}\right)=-\frac{1}{2^{k}} \sum_{m=0}^{2^{k} N_{0}-1} \nabla f\left(\mathbf{p}_{(k)}[m]\right) \cdot \mathbf{n}_{\mathrm{d}}\left(\mathbf{p}_{(k)}[m]\right)
$$


as the edge-based energy, where $\mathbf{p}_{(k)}[m]$ is the location of the $m$-th subdivision point and where $\nabla f\left(\mathbf{p}_{(k)}[m]\right)$ and $\mathbf{n}_{\mathrm{d}}\left(\mathbf{p}_{(k)}[m]\right)$ are the within-plane gradient of the image $f$ and the approximation of the normal vector, respectively. The vector $\mathbf{n}_{\mathrm{d}}=\left(\begin{array}{l}n_{\mathrm{d}, 1} \\ n_{\mathrm{d}, 2}\end{array}\right)$ is defined by

$$
\mathbf{n}_{\mathrm{d}}\left(\mathbf{p}_{(k)}[m]\right)=\left(\begin{array}{c}
2^{k}\left(p_{2(k)}[m+1]-p_{2(k)}[m]\right) \\
-2^{k}\left(p_{1(k)}[m+1]-p_{1(k)}[m]\right)
\end{array}\right)
$$

and converges to

$$
\mathbf{n}_{\mathrm{d}}\left(\mathbf{p}_{(k)}[m]\right) \underset{k \rightarrow \infty}{\longrightarrow} \mathbf{n}\left(\left.\mathbf{r}(t)\right|_{t=\frac{m}{2^{k}}}\right)=\left(\begin{array}{c}
\left.\dot{r}_{2}(t)\right|_{t=\frac{m}{2^{k}}} \\
-\left.\dot{r}_{1}(t)\right|_{t=\frac{m}{2^{k}}}
\end{array}\right),
$$

where $\mathbf{n}(\mathbf{r})$ is the vector normal to the curve $\mathbf{r}$. The main advantage of using (6) instead of only using the image gradient is that (6) incorporates information about the directionality in its expression through the vector $\mathbf{n}_{\mathrm{d}}$. This allows the snake to discriminate on which side of an object it is located (e.g., inside or outside of an object).

The region-based energy that we propose discriminates an object from its background by building a curve $\mathbf{r}_{\lambda}$ around the snake $\mathbf{r}$, obtained by dilating it by a factor $\sqrt{2}$ with respect to its center of gravity. Then, the contrast is maximized between the intensity of the data averaged over the surface $\Omega$ enclosed by the curve $\mathbf{r}$, and the intensity of the data averaged over the shell $\Omega_{\lambda} \backslash \Omega$, where $\Omega_{\lambda}$ is the surface enclosed by the curve $\mathbf{r}_{\lambda}$. Note that $\Omega \subset \Omega_{\lambda}$ and $\left|\Omega_{\lambda}\right|=2|\Omega|$. The region-based energy is expressed as

$$
\begin{aligned}
E_{\text {region }}\left(f, \mathbf{p}_{(k)}\right)= & \frac{1}{2^{k}\left|\Sigma\left(\mathbf{p}_{(k)}\right)\right|} \\
& \times\left(2 \sum_{m=0}^{2^{k} N_{0}-1} g_{1}\left(\mathbf{p}_{(k)}[m]\right) n_{\mathrm{d}, 1}\left(\mathbf{p}_{(k)}[m]\right)\right. \\
& \left.-\sum_{m=0}^{2^{k} N_{0}-1} g_{1}\left(\mathbf{p}_{\lambda(k)}[m]\right) n_{\mathrm{d}, 1}\left(\mathbf{p}_{\lambda(k)}[m]\right)\right),
\end{aligned}
$$

where $\mathbf{p}_{\lambda(k)}$ is the sequence of subdivision points that describes the curve $\mathbf{r}_{\lambda}$ and $g_{1}$ is the pre-integrated image along the first dimension defined by $g_{1}\left(p_{1}, p_{2}\right)=\int_{-\infty}^{p_{1}} f\left(\tau, p_{2}\right) \mathrm{d} \tau$. We define $\Sigma\left(\mathbf{p}_{(k)}\right)$ as

$$
\Sigma\left(\mathbf{p}_{(k)}\right)=\frac{1}{2^{k}} \sum_{m=0}^{2^{k} N_{0}-1} p_{1(k)}[m] n_{\mathrm{d}, 1}\left(\mathbf{p}_{(k)}[m]\right) .
$$

The image $g_{1}$ is precomputed and stored in a lookup table, which dramatically speeds up the computation of the algorithm.

Proposition 2: As $k \rightarrow \infty$, the energies defined by (6), (8), and (9) converge to

$$
E_{\text {edge }}\left(f, \mathbf{p}_{(k)}\right) \underset{k \rightarrow \infty}{\longrightarrow}-\int_{0}^{N_{0}} \nabla f(\mathbf{r}(t)) \cdot \mathbf{n}(\mathbf{r}(t)) \mathrm{d} t
$$

and

$$
\begin{aligned}
E_{\text {region }}\left(f, \mathbf{p}_{(k)}\right) \underset{k \rightarrow \infty}{\longrightarrow} \frac{1}{|\Sigma|}( & \iint_{\Omega} f(\mathbf{r}) \mathrm{d} r_{1} \mathrm{~d} r_{2} \\
& \left.-\iint_{\Omega_{\lambda} \backslash \Omega} f(\mathbf{r}) \mathrm{d} r_{1} \mathrm{~d} r_{2}\right),
\end{aligned}
$$

with

$$
\Sigma\left(\mathbf{p}_{(k)}\right) \underset{k \rightarrow \infty}{\longrightarrow} \Sigma=\iint_{\Omega} \mathrm{d} r_{1} \mathrm{~d} r_{2},
$$

where $\Omega$ and $\Omega_{\lambda}$ are the surfaces enclosed by the curve $\mathbf{r}$ and $\mathbf{r}_{\lambda}$, respectively, and $\Sigma$ is the signed area enclosed by the contour $\mathbf{r}$.

These are the standard energies given in [32] and [33]. The proof of Proposition 2 is given in Appendix D.

\section{Multiresolution Approach}

The segmentation outcome, when using active-contour models, depends on the initialization of the snake. A larger basin of attraction allows for a rougher initialization. With common singleresolution segmentation algorithms, a tradeoff has to be made between the desired accuracy and the amount of blurring one applies to an image. Blurring enlarges the basin of attraction but also decreases the resolution of an object, which in turn affects the quality of the delineation. Multiresolution approaches are powerful methods to speed up the optimization process and improve robustness. Existing methods mainly rely on the construction of an image pyramid, where the active contour is upsampled from a coarse scale to a finer scale of the image [34]-[36]. One limitation of those methods is that the object to segment may not have the same topology on the coarsest and finest images. In this section, we present a multiresolution approach which is inherent to the iterative process of subdivisions. The subdivision snake has the advantage that the resolution of the representation can be adapted to the resolution of the object to be segmented. The number of subdivision points used to describe the snake and to determine its energies according to Section III-B is controlled by the number $k$ of subdivisions. If fewer points are used, the optimization is faster. We exploit this multiresolution property both to enlarge the basin of attraction and to accelerate the optimization.

Algorithm: We apply $k$ successive lowpass filters $G_{k}$ to the original image to obtain $k$ smoothed images $f_{k}$. The snake is first optimized on the coarsest image $f_{1}$ that corresponds to the lowest resolution and, hence, the structure of interest only contains few details. The initialization on $f_{1}$ can be very rough because the blurring enlarges the basin of attraction. The snake is optimized on $f_{1}$ and is then used as initialization at the next resolution level on $f_{2}$. The process continues until the optimization reaches the finest resolution level that corresponds to the original image $f$. Because the smoothed images contain fewer details and less noise than the original one the snake is more robust to initial conditions. The subdivision scheme allows us to adapt the number of subdivision points describing the curve $\mathbf{r}$ to the level of detail in the image. Thus, we start with few subdivision points (i.e., one subdivision step), which allows for fast optimization. At each subsequent iteration of 
TABLE I

Multiresolution Algorithm

Input: Image $f$, low-resolution snake encoded by $\mathbf{p}_{(0)}$

$\mathbf{p}_{(1)}=\mathbf{p}_{(0)_{\uparrow_{2}}} * h$

For $k$ iterations $(k \geq 1)$ :

compute: image $f_{k}=f * G_{k}$

optimize: $\mathbf{p}_{(0)_{\text {opt }, \mathbf{k}}}=\underset{\mathbf{p}_{(0)} \arg \min }{\arg } E_{\text {snake }}\left(f_{k}, \mathbf{p}_{(k)}\right)$

$$
\mathbf{p}_{(k)_{\mathrm{opt}}}=\mathbf{p}_{(0)_{\mathrm{opt}, \mathrm{k} \uparrow_{2} k}} * h_{0 \rightarrow k}
$$

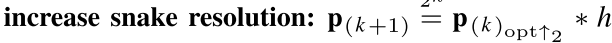

until: high-resolution segmentation on the original image $f$

the multiresolution algorithm, we keep constant the number of control points and increase the density of the subdivision points. The pseudo-code in Table I describes this algorithm. Note that the position of the control points $\mathbf{p}_{(0)}$ changes after each optimization. We denote by $\mathbf{p}_{(0)_{\mathrm{opt}, \mathrm{k}}}$ the sequence describing the optimized control points at iteration $k$. The images $f_{k}$ and their pre-integrated versions are pre-computed, which accelerates the segmentation process and decreases the memory requirements.

\section{Design of Subdivision Schemes}

When choosing or designing a subdivision mask to construct the active contour model, there are three important properties to consider. The first defines its capability to perfectly reproduce specific shapes, such as polynomial or trigonometric curves. The second is whether the control points interpolate the curve or not. The third is the support of the mask, given by the number of its non-zero elements. This can affect the optimization and, generally, a short mask is preferred over a large one. In practice, a tradeoff between the advantages and limitations regarding these properties has to be made. The purpose of this section is to offer guidance on the choice of the subdivision mask. We discuss the two most interesting families: the Deslauriers-Dubuc and the minimum-support subdivision schemes.

\section{A. Generation of Polynomials}

Proposition 3 gives a criterion that a subdivision scheme must verify to generate polynomials.

Proposition 3: (Conti and Hormann [24, eq. (7)]) A subdivision scheme generates polynomials up to degree $(L-1)$ if the $z$-transform of the subdivision mask takes the form

$$
H(z)=(1+z)^{L} B(z),
$$

where $B(z)$ is a Laurent polynomial with $B(1)=\frac{1}{2^{L-1}}$.

\section{B. Deslauriers-Dubuc Subdivision}

The Deslauriers-Dubuc subdivision scheme is convergent and interpolating [37], [38]. It reproduces polynomials up to degree $(L-1)$ [14], [39], [40]. The mask has a support of size $2(L-1)+1$ and is computed by solving the system [19], [41]

$$
\left\{\begin{array}{l}
H(z)+H(-z)=2 \\
H(z)=R(z) Q(z)
\end{array}\right.
$$

where $R(z)=(1+z)^{L}$ and $Q(z)$ is the shortest-possible polynomial. We solve (10) using Bézout's theorem and we obtain

$$
H(z)=(-1)^{\frac{L}{2}}\left(1-z^{2}\right)^{L} z^{-L}\left(\sum_{q=1}^{L} \frac{(-1)^{q} a_{q}}{(z-1)^{q}}\right),
$$

where $\left\{a_{q}\right\}_{q \in[1 \ldots L]}$ are the coefficients of the simple-fraction decomposition

$$
\frac{2(-1)^{\frac{L}{2}} z^{L}}{\left(z^{2}-1\right)^{L}}=\sum_{q=1}^{L} a_{q}\left(\frac{1}{(z+1)^{q}}+\frac{(-1)^{q}}{(z-1)^{q}}\right) .
$$

Example-Reproduction of Third-Degree Polynomials: We now focus on the particular case when $L=4$. It corresponds to the well-known subdivision scheme introduced by Deslauriers and Dubuc in [37] that reproduces polynomials up to degree 3. The corresponding subdivision mask $h$ has a support of size 7 and is defined by

$$
h[m]= \begin{cases}-\frac{1}{16}, & |m|=3 \\ 0, & |m|=2 \\ \frac{9}{16}, & |m|=1 \\ 1, & m=0 \\ 0, & \text { otherwise }\end{cases}
$$

\section{Minimum-Support Subdivision Scheme}

The minimum-support subdivision scheme has the property to generate polynomials with the shortest mask. However, it is not interpolating, meaning that the control points do not lie on the limit curve, in which case it will be less intuitive for the user to interact with the curve. The mask associated to the scheme that generates polynomials up to degree $(L-1)$ is defined as

$$
H(z)=\frac{1}{2^{L-1}}(1+z)^{L}
$$

and has a support of size $L+1$ [42].

Example-Shortest Generation of Third-Degree Polynomials: In this example, we construct a minimum-support subdivision scheme that generates polynomials up to degree 3 . The corresponding mask is of size 5 and is defined by

$$
H(z)=\frac{1}{8}+\frac{1}{2} z+\frac{3}{4} z^{2}+\frac{1}{2} z^{3}+\frac{1}{8} z^{4} .
$$

\section{Design of Non-Stationary Subdivision Schemes}

The subdivision schemes that we have described so far are called stationary, meaning that the subdivision mask $h$ is the same at each iteration $k$. A subdivision scheme is called non-stationary if the subdivision mask $h_{k}$ is different at each iteration $k$, with the rest of the procedure being the same as in Section II.B. Non-stationary subdivision schemes are 
required to reproduce exponential polynomials, which allows to construct trigonometric functions. The refinement rule is now

$$
\mathbf{p}_{(k)}=h_{k} * \mathbf{p}_{(k-1) \uparrow_{2}},
$$

where $h_{k}$ is the subdivision mask at the $k$ th iteration. The relation between the periodic sequence $\mathbf{p}_{(k)}$ at the $k$ th iteration and the control points $\mathbf{p}_{(0)}$ is still defined by (2) but $h_{0 \rightarrow k}$ is now computed by

$$
h_{0 \rightarrow k}=h_{1_{\uparrow^{k-1}}} * h_{\uparrow_{\uparrow^{k-2}}} * \cdots * h_{k-1_{\uparrow_{2}}} * h_{k} .
$$

If we set $h=h_{k}$, we recover all the formulas of the stationary scheme. Furthermore, every convergent stationary subdivision scheme verifies the property of affine invariance stated in Definition 1 (see Proposition 1). In the non-stationary setting, however, it must be verified case by case [24].

\section{A. Generation of Exponential Polynomials}

We define $\boldsymbol{\gamma}=\left(\gamma_{1}, \gamma_{2}, \ldots, \gamma_{L}\right)$ and denote by $L_{m}$ the multiplicity of the element $\gamma_{m} \in \gamma$, for $m=1, \ldots, L$. A non-stationary subdivision scheme is said to generate exponential polynomials if it generates the whole family $\left\{\mathrm{e}^{\gamma_{m} t} t^{n}\right\}_{n \in\left[0 \ldots L_{m}-1\right]}$. In this case, the subdivision mask at the $k$ th iteration is characterized by $\boldsymbol{\gamma}_{k}=\frac{\gamma}{2^{k}}$ and its $z$-transform is denoted by $H_{k}^{\gamma}$.

\section{B. Generation of Trigonometric Functions}

The generation of trigonometric functions allows one to efficiently construct a scheme that is capable of generating circles and ellipses which are useful structures in the context of segmentation in bioimaging. We now present a criterion that a (non-stationary) subdivision scheme must verify to generate trigonometric functions.

Proposition 4: (Romani [43, Proposition 2]) A nonstationary subdivision scheme perfectly generates ellipses if the $z$-transform of the subdivision mask at the $k$ th iteration verifies

$$
H_{k}(z)=(1+z)\left(1+\mathrm{e}^{\frac{j}{2^{k} N_{0}}} z\right)\left(1+\mathrm{e}^{\frac{-\mathrm{j} 2 \pi}{2^{k} N_{0}}} z\right) Q_{k}(z),
$$

where $Q_{k}(z)$ is a polynomial in $z$.

That means that the subdivision scheme has to generate exponential polynomials and that $\left(0, \frac{\mathrm{j} 2 \pi}{N_{0}}, \frac{-\mathrm{j} 2 \pi}{N_{0}}\right) \subset \boldsymbol{\gamma}$. In the following we provide two examples of ellipse-generating subdivision schemes: the non-stationary Deslauriers-Dubuc and the non-stationary minimum-support subdivision schemes.

\section{Non-Stationary Deslauriers-Dubuc Subdivision Scheme}

The non-stationary Deslauriers-Dubuc subdivision scheme is interpolating and capable of reproducing the exponential polynomials defined in Section V-A [19], [41], [44]. As for the stationary case, the mask at the $k$ th iteration has a support of size $2(L-1)+1$ and is obtained by solving

$$
\left\{\begin{array}{l}
H_{k}^{\gamma}(z)+H_{k}^{\gamma}(-z)=2 \\
H_{k}^{\gamma}(z)=R^{\gamma_{k}}(z) Q_{k}(z),
\end{array}\right.
$$

where $R^{\gamma}(z)=\prod_{m=1}^{L}\left(1+\mathrm{e}^{\gamma_{m}} z\right), \gamma_{k}=\frac{\gamma}{2^{k}}$, and $Q_{k}(z)$ is a polynomial in $z$. Vonesch et al. [41] extensively studied this scheme and proposed simplified solutions to solve (11) by applying Bézout's identity

$$
C_{k}(Z) D_{k}(Z)+C_{k}(-Z) D_{k}(-z)=2
$$

where $Z=\frac{z+z^{-1}}{2}, C_{k}(Z)=z^{-\frac{L}{2}} R^{\gamma_{k}}(z)$, and $D_{k}(Z)=$ $z^{\frac{L}{2}} Q_{k}(z)$. The shortest polynomial $D_{k}(Z)$ is given by

$$
D_{k}(Z)=\left(\sum_{q=1}^{K} \sum_{s=1}^{L_{q}} \frac{(-1)^{s} a_{q, s}}{\left(Z+Z_{q}\right)^{s}}\right) C_{k}(-Z),
$$

where $K<L$ is the number of different elements of $\boldsymbol{\gamma}$, $\left\{Z_{q}\right\}_{q \in[1 \ldots K]}$ are the roots of $C_{k}(Z)$ with multiplicity $L_{q}$, and $\left\{a_{q, s}\right\}_{q \in[1 \ldots K], s \in\left[1 \ldots L_{q}\right]}$ are the coefficients of the simplefraction decomposition

$$
\frac{2}{C_{k}(-Z) C_{k}(Z)}=\sum_{q=1}^{K} \sum_{s=1}^{L_{q}} a_{q, s}\left(\frac{1}{\left(Z-Z_{q}\right)^{s}}+\frac{(-1)^{s}}{\left(Z+Z_{q}\right)^{s}}\right) .
$$

Example-Ellipse-Reproducing Scheme: We construct a non-stationary Deslauriers-Dubuc subdivision scheme that is capable of reproducing ellipses. Therefore, we want to be able to construct trigonometric functions. According to Proposition $4,\left(0, \frac{\mathrm{j} 2 \pi}{N_{0}}, \frac{-\mathrm{j} 2 \pi}{N_{0}}\right) \subset \boldsymbol{\gamma}$. Moreover, it was shown in [41] that the elements of $\gamma$ must come in complexconjugate pairs and that, if 0 is an element of $\boldsymbol{\gamma}$, then it must have even multiplicity. Hence, $\boldsymbol{\gamma}=\left(0,0, \frac{2 \mathrm{j} \pi}{N_{0}},-\frac{2 \mathrm{j} \pi}{N_{0}}\right)$. The mask at iteration $k$ is of size 7. By solving (11), for $N_{0}=4$, we obtain the scheme

$$
h_{k}[m]= \begin{cases}-\frac{2^{k}}{-1} & |m|=3 \\ \frac{\left(1+\sqrt[2^{k+1}]{-1}\right)^{2}\left(1+\sqrt[2^{k}]{-1}\right)}{2\left(1+\sqrt[2^{k}]{-1}\right)^{2}}, & |m|=1 \\ 1, & m=0 \\ 0, & \text { otherwise. }\end{cases}
$$

Note that, when $k \rightarrow \infty$, the mask $h_{k}$ converges towards the stationary Deslauriers-Dubuc scheme given in Section IV-B which reproduces polynomials of degree up to 3 .

\section{Non-Stationary Minimum-Support Subdivision Scheme}

The non-stationary minimum-support subdivision scheme generates exponential polynomials defined in Section V-A with the shortest mask [45]. It has a support of size $L+1$ and is given by

$$
H_{k}^{\gamma}(z)=\frac{1}{2^{L-1}} \prod_{m=1}^{L}\left(1+\mathrm{e}^{\frac{\gamma_{m}}{2^{k}}} z\right) .
$$

Example-Shortest Ellipse-Generating Scheme: We construct a non-stationary minimum-support subdivision scheme that 


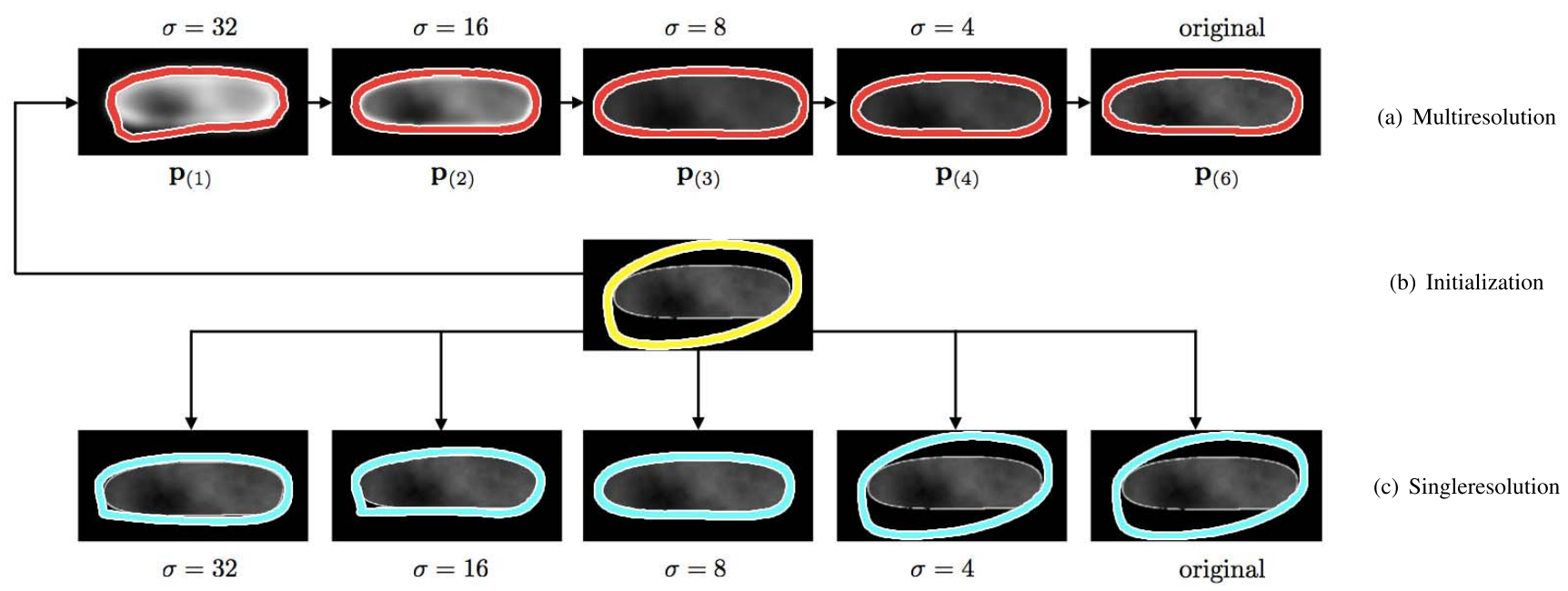

Fig. 4. Comparison of the accuracy of the segmentation between the multiresolution subdivision snake and the parametric singleresolution snake. Both snakes generate polynomials of degree up to 2. (a) Evolution of the subdivision snake during the six-level multiresolution process. The last illustration shows the final segmentation on the original image. (b) Initialization. (c) Several segmentation results obtained with the parametric singleresolution snake for different blurred versions of the original test image.

is capable of generate ellipses. Therefore, we choose $\boldsymbol{\gamma}=\left(0, \frac{2 \mathrm{j} \pi}{N_{0}},-\frac{2 \mathrm{j} \pi}{N_{0}}\right)$. By imposing the affine invariance of Definition 1, the subdivision mask at iteration $k$ is of size 4 and is given by $\operatorname{sinc}^{-2}\left(\frac{1}{N_{0}}\right) H_{k}^{\gamma}(z)$, where

$$
\begin{aligned}
H_{k}^{\gamma}(z)=\frac{1}{4}( & +\left(1+\mathrm{e}^{\frac{-2 \mathrm{j} \pi}{2^{k} N_{0}}}+\mathrm{e}^{\frac{2 j \pi}{2^{k} N_{0}}}\right) z \\
& \left.+\left(1+\mathrm{e}^{\frac{-2 \mathrm{j} \pi}{2^{k} N_{0}}}+\mathrm{e}^{\frac{2 \mathrm{j} \pi}{2^{k} N_{0}}}\right) z^{2}+z^{3}\right) .
\end{aligned}
$$

\section{EXPERIMENTS AND VALIDATION}

In this section, we compare the proposed multiresolution snake to parametric singleresolution snakes [29]. We first test the robustness w.r.t. initial conditions and, in a second step, we measure its robustness w.r.t. noise as well as its ability to segment objects of varying sizes in an image. Finally, we illustrate applications on real data where the ground truth is not available. For each experiment the optimization of the snakes is carried out by a Powell-like line-search method [46].

\section{A. Accuracy and Robustness to Initial Conditions}

We carry out two experiments in which we compare the multiresolution subdivision snake to a parametric singleresolution snake based on quadratic B-splines as described in [29]. In order to compare snakes with the same reproduction properties, the subdivision snake is constructed with a minimum-support subdivision scheme that generates polynomials of degree up to 2 (see Section IV-C).

In the first experiment, we test the accuracy of the segmentation. We use the Jaccard index to measure the overlap between the segmentation result and the ground truth. For two sets $A$ and $B$, it is defined as

$$
J=\frac{|A \cap B|}{|A \cup B|} .
$$

Clearly, $0 \leq J \leq 1$, and the maximum overlap is described by $J=1$. We created a test image of $854 \times 768$ pixels that
TABLE II

JACCARD INDICES FOR SEGMENTATION OBTAINED WITH THE SINGLERESOLUTION AND SUBDIVISION SNAKES, BOTH Generating Polynomials of Degree UP to 2

\begin{tabular}{cccc}
\hline \hline & $\sigma$ [pixel] & $J$ & Result \\
\hline & 32 & 0.803 & fail \\
Singleresolution & 16 & 0.860 & fail \\
snake & 8 & 0.950 & succeed \\
& 4 & 0.544 & fail \\
& 2 & 0.544 & fail \\
\hline Subdivision snake & 0 & 0.544 & fail \\
\hline \hline
\end{tabular}

simulates realistic conditions in fluorescence microscopy (see Figure 4(b)), including noise. It shows a rod-shaped cell representative of a Schizosaccharomyces pombe (S. pombe) [47]. We then blurred the test image with five Gaussian kernels having different standard deviations $\sigma$, which are given in the first column of Table II. Four resulting images are shown in Figure 4(a). The higher the standard deviation, the fewer details are present in the filtered image. The initialization of the snakes was drawn manually with $N_{0}=8$ control points (Figure 4(b)). Its overlap with the actual structure corresponds to the Jaccard index $J=0.544$. First, we optimized the subdivision snake using the multiresolution algorithm described in Section III-C. At each iteration we did one subdivision step corresponding to a multiplication by a factor of 2 , starting with $2 N_{0}=16$ subdivision points. The curve evolves guided by an edge energy. The optimized contours at different levels of the multiresolution algorithm are shown in Figure 4(a). We compared the final segmentation to the ground truth of the synthetic data; the corresponding Jaccard index is given in Table II. We consider that a snake succeeds in segmenting the structure of interest if $J \geq 0.95$. We then independently optimized the singleresolution snake with an edge energy on the six images (the five blurred images and the original one) using the same initialization. Results are shown in Figure 4(c) and the corresponding Jaccard indices are given in Table II. 


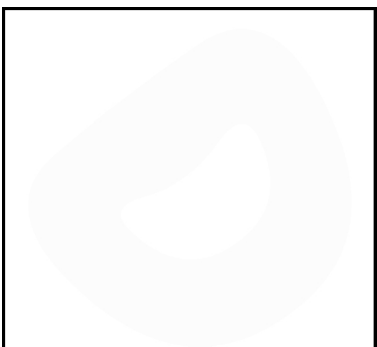

(a) $\sigma=0$

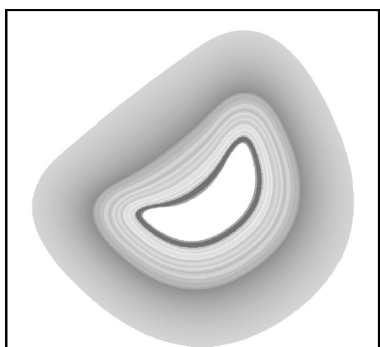

(b) $\sigma=10$

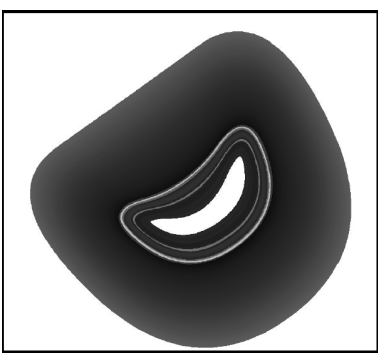

(c) Multiresolution

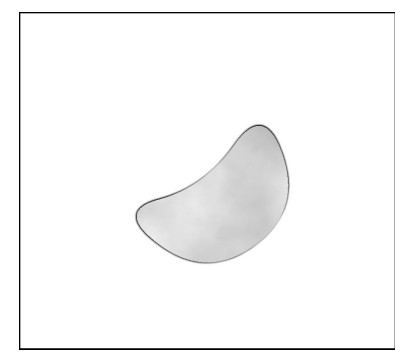

(d) Test image

Fig. 5. Comparison of the basin of attraction of the multiresolution subdivision snake versus the parametric singleresolution snake using an edge energy. Basins of attraction of the singleresolution snake ((a) and (b)) were obtained for the original image and for a blurred version $(\sigma=10)$. (c) Basin of attraction of the multiresolution subdivision snake obtained on the original image (d).

The segmentation succeeded only on the smoothed image corresponding to $\sigma=8$. The singleresolution snake is able to segment the structure of interest only on a smoothed image because the basin of attraction is too narrow otherwise for the edge energy. The variance of the Gaussian filter has to be well-chosen according to the initialization. We conclude that the multiresolution approach improves the accuracy of the segmentation. This result is explained by the fact that the multiresolution is initialized on the coarsest image with reduced details and a large basin of attraction. By adapting the resolution of the subdivision snake to the image details, it is able to converge to the structure to segment on the original image.

In the second experiment, we evaluate the impact of the multiresolution approach on the robustness of the snake w.r.t. the initialization. For this experiment, we generated another test image (Figure $5(\mathrm{~d})$ ) of $854 \times 768$ pixels of a sickle cell [48] acquired through fluorescence microscopy. We compared the basin of attraction of both the singleresolution and the multiresolution subdivision snakes using $N_{0}=6$ control points. Each basin of attraction was computed as follows: a rough approximation of the goldstandard was constructed. This shape was rescaled to construct several initial positions of the snake. We optimized the snake using an edge energy. For each segmentation result, we computed the Jaccard index and associated a grayscale value to $J$ where white corresponds to $J=0$ and black to $J=1$. We generated an image where each initialization was drawn with the color corresponding to the Jaccard index of the corresponding segmentation result. For the singleresolution snake, we realized this experiments on two images: the original one and a smoothed version with $\sigma=10$, where the results are shown in Figure 5(a) and (b), respectively. For the subdivision snake, we used the multiresolution approach on the original image. The result is given in Figure 5(c). The white regions in the images showing the basin of attraction correspond to positions that were not considered for initialization, including the boundary of the shape to segment. Note that the average Jaccard values inside the shape to segment appear to be less uniform than outside. This can be attributed to the two following reasons: First, as seen on the original image (Figure 5(d)), the outside of the shape is completely uniform in intensity while the inside of the shape exhibits variations in pixel values. Snakes evolving from
TABLE III

JACCARD INDICES FOR THE SEgMENTATION OF NOISY DATA

\begin{tabular}{cc}
\hline \hline SNR $[\mathrm{dB}]$ & $J$ \\
\hline-7.83 & 0.990 \\
-13.80 & 0.987 \\
-16.60 & 0.987 \\
-17.82 & 0.984 \\
\hline \hline
\end{tabular}

outside of the object therefore encounter no risk of getting diverted from their target due to variations of pixel intensities. Snakes which start to deform from the inside of the shape are, however, evolving on a nonuniform region and are more likely to get trapped into local energy minima. Second, for a given number of control points, smaller snakes tend to diverge more easily than larger ones. This effect is simply due to the fact that, if their number is fixed, control points are physically closer in smaller shapes. During the optimization process and as the control points are moved, it becomes therefore more likely for the snake to get entangled. In the present experiment, initial shapes inside the object to segment are smaller than the ones outside the object, and optimization results tend to get more unstable due to the enhanced risk of entanglement. We observe that the singleresolution snake is very sensitive to the initialization. On the contrary, the subdivision snake leads to accurate segmentation even for initializations far from the object to segment.

\section{B. Robustness With Respect to Noise}

As further test of robustness, we performed segmentation on data with different levels of additive white Gaussian noise. We used the multiresolution subdivision snake constructed with the minimum-support subdivision scheme generating polynomials of degree up to 2 and $N_{0}=8$ control points (Figure 6(b)). Signal-to-noise ratios (SNRs) corresponding to a given noise level and associated Jaccard indices were computed. We used a pixelwise SNR that compares the noisy image and the ground truth image. The results for the test image of Section VI-A are summarized in Table III and Figure 6(a). The initial overlap of the snake with the ground truth corresponds to $J=0.593$. For all cases, we obtained $J>0.95$. 

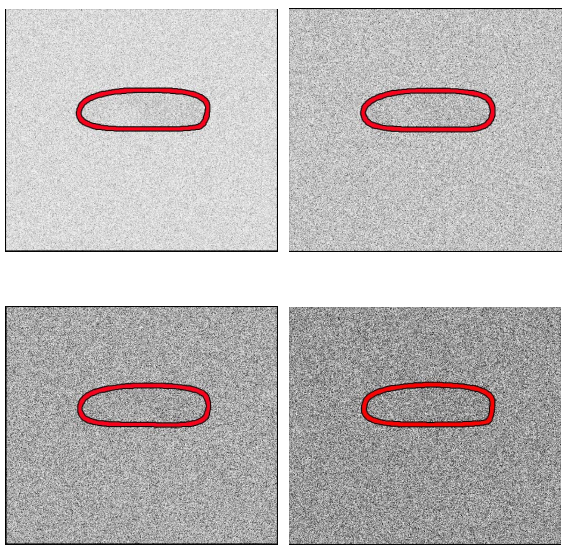

(a)

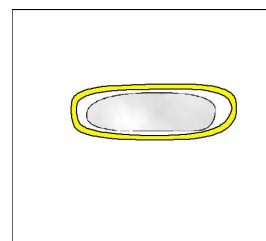

(b)

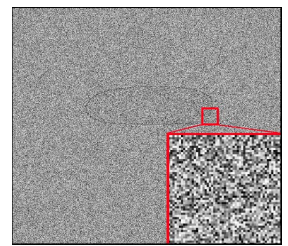

(c)
Fig. 6. Robustness w.r.t. noise of the multiresolution subdivision snake (a) Top left: $\mathrm{SNR}=-7.83 \mathrm{~dB}$; top right: $\mathrm{SNR}=-13.80 \mathrm{~dB}$; bottom left: SNR $=-16.60 \mathrm{~dB}$; bottom right: SNR $=-17.82 \mathrm{~dB}$. (b) Initialization. (c) Close-up of a boundary region between the test rod-shape and its background, $\mathrm{SNR}=-17.82 \mathrm{~dB}$.

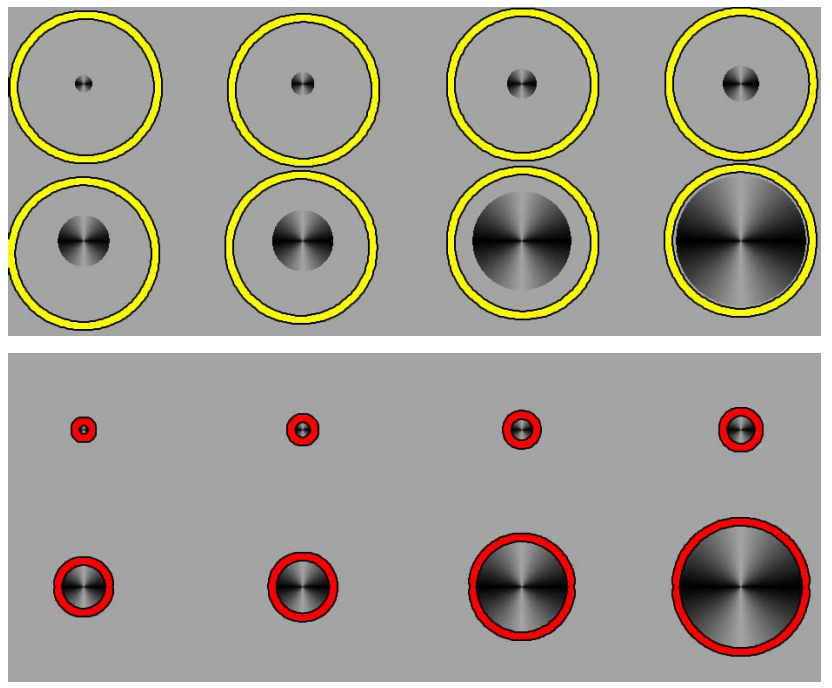

Fig. 7. Segmentation of circles with different radii using the ellipsereproducing Deslauriers-Dubuc subdivision scheme. Top: initializations; bottom: segmentation results.

\section{Segmentation of Objects of Varying Sizes}

The multiresolution algorithm for segmentation presented in Section III-C suggests that the approach is very robust to initialization. To verify this property, we created a test image of size 5,500 $\times 2,700$ pixels (Figure 7), which is composed of eight circular cells of different sizes. The initializations correspond to circles with a radius of 461 pixels centered in each cell (Figure 7, top). By adjusting the variance of the lowpass filters to the smallest structure present in the image, we were able to segment all the cells. We used a multiresolution subdivision snake based on the ellipse-reproducing Deslauriers-Dubuc scheme presented in Section V-C. Results are shown in Figure 7 (bottom) and the corresponding Jaccard indices are presented in Table IV (first line). Each structure was accurately segmented with $J \geq 0.95$.

\section{Real Data}

We illustrate the behaviour of the proposed snake on real data. In this context, the ground truth is unknown and we have
TABLE IV

JACCARD INDices FOR THE SEgMENTATION OF CiRCles OF VARIOUS SizES ObTained With the Stationary and THE NON-STATIONARY DESLAURIERS-DUbuC SCHEMES

\begin{tabular}{c|cccc}
\hline \hline $\begin{array}{c}\text { Radius [pixels] } \\
\text { Reproducing scheme }\end{array}$ & 55 & 75 & 95 & 115 \\
\hline $\begin{array}{c}\text { Ellipses } \\
\text { Polynomials of degree up to 3 }\end{array}$ & 0.992 & 0.994 & 0.995 & 0.996 \\
\hline \hline Radius [pixels] & 165 & 195 & 315 & 415 \\
\hline \hline Reproducing scheme & & & & \\
\hline Ellipses & 0.996 & 0.996 & 0.997 & 0.998 \\
Polynomials of degree up to 3 & 0.756 & 0.777 & 0.771 & 0.765 \\
\hline \hline
\end{tabular}

to rely on qualitative assessments to validate the accuracy of the segmentation. We applied our multiresolution subdivision snake, constructed with the non-stationary minimum-support subdivision scheme that generates ellipses (Section V-D), to four microscopic images (Figure 8(b)). These images are challenging because of the presence of noise and of objects with different sizes. Moreover, shapes can be close to each other. They represents elliptic cells (top left, inverted contrast), rod-shaped cells of $S$. pombe (top right), circular cells (bottom left), and a sickle cell (bottom right). The initializations are shown in Figure 8(a). The qualitative assessment of the segmentation yields satisfactory results. We used both the edge and region energies and the average time to delineate one cell was less than 0.2 seconds on a $1.7 \mathrm{GHZ}$ processor with 8 GB RAM.

Note that, as the principal motivation for our work is the segmentation of biological images, it was important trough those experiments to show that our model can reproduce circular or elliptic shapes. However, the reproduction properties of the presented schemes are not restricted to those shapes. More complex shapes can be segmented by increasing the number of control points.

\section{DISCUSSION}

\section{A. Guidelines for the Choice of the Subdivision Scheme}

Minimum-Support vs. Deslauriers-Dubuc Subdivision Schemes: The computation of the snake energy and the speed of the optimization algorithm is related to the length of the support $N$ of the subdivision mask. More precisely, the complexity when calculating the subdivision points (2) is $\mathcal{O}\left((N-1)^{k}\right)$. Therefore, the fastest algorithm is obtained using minimum-support subdivision schemes. In return, the Deslauriers-Dubuc subdivision is interpolating. This can be an advantage if user interaction is involved, because it facilitates the editing of the curve. We present in Figure 9 an intermediate stage in the segmentation of a dividing cell. User interaction makes it possible to improve the result by moving the control points. However, the interaction is more intuitive when they lie on the curve (Figure 9(b)). Otherwise, it is difficult to know which parameter controls the part of the curve that has to be modified (Figure 9(a)).

The choice of the subdivision mask ultimately depends on the application: for an automatic method, we suggest to use a minimum-support subdivision scheme; whereas, 


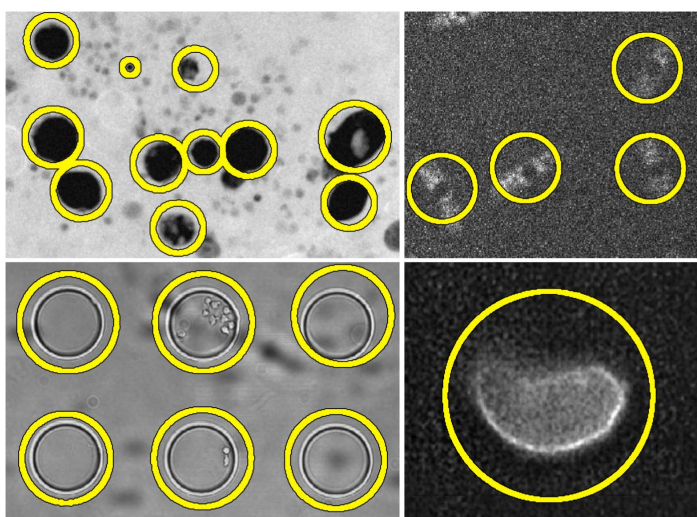

(a)

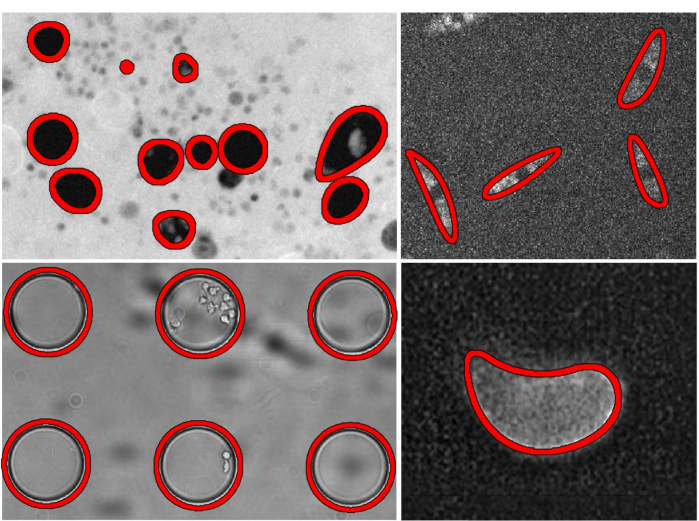

(b)

Fig. 8. Segmentation of real data using multiresolution subdivision snakes constructed with the non-stationary minimum-support subdivision scheme that generates ellipses. (a) Initial contours of the snakes. (b) Segmentation of: elliptic cells (top left); S. pombes (top right); circular cells (bottom left), and a sickle cell (bottom right).

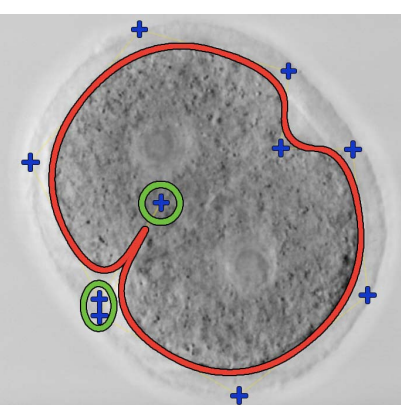

(a)

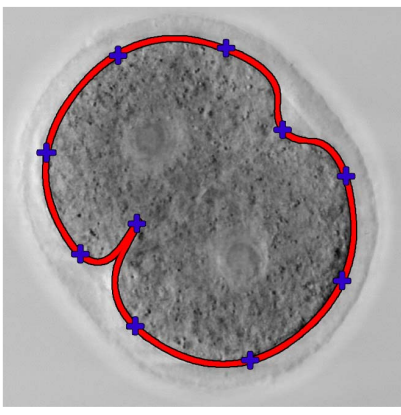

(b)

Fig. 9. User-friendly interaction according to the interpolation property of the subdivision scheme. (a) Non-interpolating control points. (b) Interpolating control points. Blue crosses: control points; red curve: snake; green circles: control points for which it is difficult to know which part of the curve they control. Source: http://www.cellimagelibrary.org/images/35450/.

when one would like to benefit from friendly user interactions, it is preferable to use a Deslauriers-Dubuc subdivision scheme.

Stationary vs. Non-Stationary Subdivision Schemes: Nonstationary subdivision schemes are somewhat more complicated than stationary ones because the subdivision mask is different at each iteration. Their main advantage lies in their capability to reproduce cosinus and sinus, which allows for an efficient construction of ellipses and circles. In biomedical imaging, circular or elliptic structures are often encountered. It is therefore desirable for the snake to be able to reproduce these shapes. The non-stationary schemes presented in Sections V-C and V-D reproduce ellipses with the minimum number of control points $N_{0}=3$, whereas the reproduction is only approximated with the stationary schemes for $N_{0}<+\infty$. As the speed of the algorithm scales with the number of control points, it is preferable to use a non-stationary subdivision schemes with few control points to segment elliptic structures. To illustrate this property, we computed the error when approximating a circle as a function of $N_{0}$ with the stationary Deslauriers-Dubuc subdivision scheme. In Figure 10, we see that the error decreases as $N_{0}$ increases. However, a large

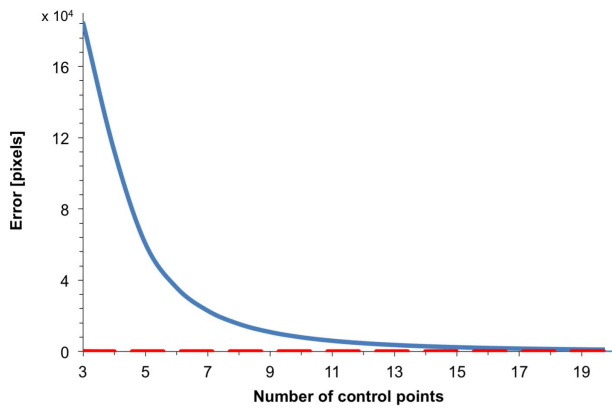

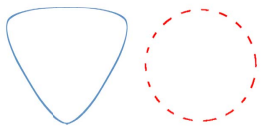

(b) $N_{0}=3$

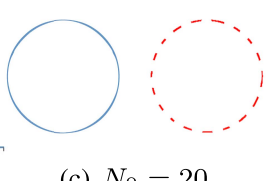

(c) $N_{0}=20$ (a)

Fig. 10. Approximation of trigonometric curves with the stationary (blue solid line) and non-stationary (red dashed line) Deslauriers-Dubuc subdivision schemes. (a) Evolution of the approximation error as a function of the control points. Approximated ellipses for $N_{0}=3$ (b) and $N_{0}=20$ (c) are given for each scheme.

number of control points is needed to obtain an acceptable error. Therefore, the segmentation of circular shapes with a small number of control points $\mathbf{p}_{(0)}$ is more accurate with a non-stationary scheme. To highlight this property, we performed the same experiment as the one presented in Figure 7, using the stationary Deslauriers-Dubuc scheme that reproduces polynomials of degree up to 3 . We used the same initializations and $N_{0}=4$ control points. The results are shown in Figure 11 . We computed the Jaccard indices and compared them to the ones obtained previously with the non-stationary scheme (see Table IV). All the Jaccard indices are worse than 0.95, which is due to the fact that the stationary scheme does not approximate well circles for $N_{0}=4$.

To conclude, if the structure of interest has many details, that requires a high number of control points, then we suggest the use of stationary schemes, thereby privileging computation simplicity while preserving the accuracy of the result; otherwise, one should use a non-stationary scheme. Note that, in the particular case where the basis functions of classical parametric snakes are refinable [49], so called scaling functions, there is a connection with the proposed 


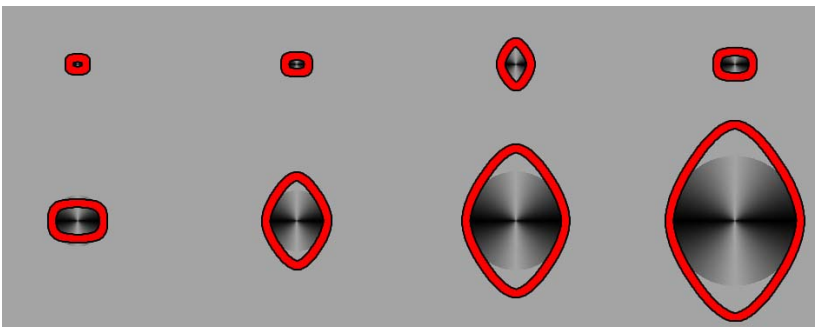

Fig. 11. Segmentation of circles of different sizes obtained with the multiresolution subdivision snake based on the stationary Deslauriers-Dubuc scheme that reproduces polynomials of degree up to 3 .

TABLE V

PROPERTIES OF THE DIFFERENT SUBDIVISION SCHEMES

\begin{tabular}{cccc}
\hline \hline Subdivision scheme & $\begin{array}{c}\text { Ellipse re- } \\
\text { production }\end{array}$ & Interpolant & $\begin{array}{c}\text { Shortest } \\
\text { mask }\end{array}$ \\
\hline Minimun-support (MS) & No & No & Yes \\
Non-stationary MS & Yes & No & Yes \\
Deslauriers-Dubuc (DD) & No & Yes & No \\
Non-stationary DD & Yes & Yes & No \\
\hline \hline
\end{tabular}

work: the discrete filters of the scaling functions can be used as subdivision masks for stationary schemes.

Summary: The properties and advantages of each subdivision scheme presented in Sections IV and V are summarized in Table V. As in biomedical imaging we often deal with elliptic structures and that biologists may need to interact with the segmentation result, we preconize the use of the non-stationary Deslauriers-Dubuc subdivision scheme.

\section{B. Choice of the Multiresolution Parameters in Practice}

Regarding the variance and the number of subdivision steps at each level of the multiresolution algorithm, we found in practice that six subdivision iterations are enough to obtain satisfactory convergence. At each resolution level, we compute one subdivision step, so that the samples of the curve are upsampled by a factor 2 . As smoothing is equivalent to a downsampling operation, we obtain the variance of the coarser lowpass filter by decreasing the resolution of the original image by a factor 2 at each iteration. Hence, we propose a multiresolution algorithm with 6 levels where the first one is characterized by $\sigma=2^{5}$ and $2 N_{0}$ subdivision points. At each iteration, the value of $\sigma$ is divided by two and one subdivision step is performed. The value of the parameters at each step are summarized in Table VI. The choice of these parameters holds when the snake is initialized far from the object to segment. Otherwise, a smaller variance can be used for the coarsest lowpass filter but the convergence of the subdivision scheme is still required on the finest level.

\section{CONCLUSION AND SUMMARY}

We have presented the 2D generic construction of multiresolution snakes based on subdivision. The snakes approximate closed curves with arbitrary precision by iteratively refining a set of control points. We have provided several examples of explicit constructions of such snakes and discussed
TABLE VI

PARAMETERS OF THE MULTIRESOLUTION ALGORITHM

\begin{tabular}{c|cccccc}
\hline $\begin{array}{r}\text { Level } \\
\text { Parameter }\end{array}$ & 1 & 2 & 3 & 4 & 5 & 6 \\
\hline$\sigma$ & 32 & 16 & 8 & 4 & 2 & 0 \\
$\mathbf{p}_{(k)}$ & $\mathbf{p}_{(1)}$ & $\mathbf{p}_{(2)}$ & $\mathbf{p}_{(3)}$ & $\mathbf{p}_{(4)}$ & $\mathbf{p}_{(5)}$ & $\mathbf{p}_{(6)}$ \\
\hline \hline
\end{tabular}

their properties. We have shown how they should be chosen according to desired properties that depend on the structures to be segmented. We have also proposed a multiresolution algorithm to adapt the resolution of the curve to the level of details in the image. We have compared our framework to traditional parametric singleresolution snakes and shown that our snakes have a larger basin of attraction, which means that they are more robust w.r.t. initial conditions. Furthermore, the multiresolution property accelerates the optimization. We have validated our snakes on test data as well as on real bioimages. We have implemented the method described in this paper as a user-friendly open-source plugin available ${ }^{2}$ for the bioimaging platform Icy [50]. This paper is a first step on the way of designing subdivision active contours of higher dimensions.

\section{APPENDIX}

A. Derivation of $\mathbf{p}_{(k)}=h_{0 \rightarrow k} * \mathbf{p}_{(0)_{\uparrow} k}$

Using (1), we have that

$$
\mathbf{p}_{(1)}[m]=\sum_{n \in \mathbb{Z}} h[m-2 n] \mathbf{p}_{(0)}[n]
$$

and

$$
\begin{aligned}
\mathbf{p}_{(2)}[m] & =\sum_{n \in \mathbb{Z}} h[m-2 n] \mathbf{p}_{(1)}[n] \\
& =\sum_{n \in \mathbb{Z}} h[m-2 n] \sum_{q \in \mathbb{Z}} h[n-2 q] \mathbf{p}_{(0)}[q] \\
& =\sum_{q \in \mathbb{Z}}\left(\sum_{u \in \mathbb{Z}} h[m-4 q-2 u] h_{\uparrow_{2}}[2 u]\right) \mathbf{p}_{(0)}[q] \\
& =\sum_{q \in \mathbb{Z}} \underbrace{\left(h * h_{\uparrow_{2}}\right)}_{h_{0 \rightarrow 2}}[m-4 q] \mathbf{p}_{(0)}[q] .
\end{aligned}
$$

Combining (1), (12), and (13), we recursively obtain

$$
\mathbf{p}_{(k)}[m]=\sum_{n \in \mathbb{Z}} h_{0 \rightarrow k}\left[m-2^{k} n\right] \mathbf{p}_{(0)}[n],
$$

where $h_{0 \rightarrow k}$ is given by (3).

\section{B. Calculation of $\dot{\mathbf{r}}\left[\frac{m}{2^{k}}\right]$}

$$
\begin{aligned}
\left.\dot{\mathbf{r}}(t)\right|_{t=\frac{m}{2^{k}}} & =\left.\lim _{\epsilon \rightarrow 0} \frac{\mathbf{r}(t+\epsilon)-\mathbf{r}(t)}{\epsilon}\right|_{t=\frac{m}{2^{k}}} \\
& =\left.\lim _{k \rightarrow \infty} 2^{k}\left(\mathbf{r}\left(t+\frac{1}{2^{k}}\right)-\mathbf{r}(t)\right)\right|_{t=\frac{m}{2^{k}}},
\end{aligned}
$$

\footnotetext{
${ }^{2}$ The plugin will be available for Windows/Linux / Mac at http://bigwww. epfl.ch/algorithms.html.
} 
where we used $\epsilon=\frac{1}{2^{k}}$. Combining this result with (4), we obtain

$$
\left.\dot{\mathbf{r}}(t)\right|_{t=\frac{m}{2^{k}}}=\lim _{k \rightarrow+\infty} 2^{k}\left(\mathbf{p}_{(k)}[m+1]-\mathbf{p}_{(k)}[m]\right) .
$$

\section{Proof of Proposition 1}

For any convergent subdivision scheme, the $z$-transform of the subdivision mask verifies $H(z)=(1+z) B(z)$, where $B(z)$ is a Laurent polynomial with $B(1)=1$ (see Section II-C). We rewrite (3) in the $z$ domain and we obtain

$$
\begin{aligned}
H_{0 \rightarrow k}(z) & =\left(\prod_{m=0}^{k-1}\left(1+z^{2^{m}}\right)\right)(\underbrace{\prod_{m=0}^{k-1} B\left(z^{2^{m}}\right)}_{Q_{k}(z)}) \\
& =\left(\sum_{m=0}^{2^{k}-1} z^{m}\right) Q_{k}(z), \forall k>0,
\end{aligned}
$$

where $Q_{k}$ is a Laurent polynomial with $Q_{k}(1)=1$. Equation (14) is equivalent to saying that the convergent subdivision scheme associated to the subdivision mask $h_{0 \rightarrow k}$ and with a refinement factor equal to $2^{k}$ reproduces constants [24, eq. (7)]. Thus, we have that

$$
\sum_{n \in \mathbb{Z}} h_{0 \rightarrow k}\left[m-2^{k} n\right]=1, \forall m \in \mathbb{Z} .
$$

Let $\mathbf{A}$ be a $(2 \times 2)$ matrix and $\mathbf{b} \in \mathbb{R}^{2}$ be a translation vector. We calculate

$$
\begin{aligned}
\left(h_{0 \rightarrow k} *\left(\mathbf{A} \mathbf{p}_{(0)}+\mathbf{b}\right)_{\uparrow_{2^{k}}}\right)[m]= & \left(\mathbf{A} h_{0 \rightarrow k} * \mathbf{p}_{(0)_{\uparrow_{2^{k}}}}\right)[m] \\
& +\mathbf{b} \sum_{n \in \mathbb{Z}} h_{0 \rightarrow k}\left[m-2^{k} n\right] .
\end{aligned}
$$

We use (2) and (15) in (16) to obtain

$$
\left(h_{0 \rightarrow k} *\left(\mathbf{A p}_{(0)}+\mathbf{b}\right)_{\uparrow_{2^{k}}}\right)[m]=\mathbf{A p}_{(k)}[m]+\mathbf{b} .
$$

For $k \rightarrow+\infty$ in (17), we obtain

$$
\lim _{k \rightarrow \infty}\left(h_{0 \rightarrow k} *\left(\mathbf{A p}_{(0)}+\mathbf{b}\right)_{\uparrow_{2^{k}}}\right)[m]=\left.\mathbf{A r}(t)\right|_{t=\frac{m}{2^{k}}}+\mathbf{b}
$$

which corresponds to the condition on affine invariance.

\section{Proof of Proposition 2}

For this proof we first recall two theorems.

Theorem of the Riemann Sum: Let $g:[a, b] \rightarrow \mathbb{R}$ be a real function that is Riemann-integrable on $[a, b]$. The Riemann sum $R_{n}$ is defined by $R_{n}=\frac{b-a}{n} \sum_{m=0}^{n-1} g\left(m \frac{b-a}{n}\right)$ and converges to $\lim _{n \rightarrow+\infty} R_{n}=\int_{a}^{b} g(t) \mathrm{d} t$.

Green's Theorem: Let $C$ be a positively oriented, piecewisesmooth, simple closed curve in a plane and let $\Omega$ be the region bounded by $C$. If $Q$ and $M$ are functions of $\left(r_{1}, r_{2}\right)$ defined on an open region containing $\Omega$ and have continuous partial derivatives there, then

$$
\oint_{C}\left(Q \mathrm{~d} r_{1}+M \mathrm{~d} r_{2}\right)=\iint_{\Omega}\left(\frac{\partial M}{\partial r_{1}}-\frac{\partial Q}{\partial r_{2}}\right) \mathrm{d} r_{1} \mathrm{~d} r_{2},
$$

where the path of integration along $C$ is counterclockwise.
For the edge energy, using (4), (6), and (7), we obtain

$$
\lim _{k \rightarrow \infty} E_{\text {edge }}\left(f, \mathbf{p}_{(k)}\right)=\underbrace{-\lim _{k \rightarrow \infty} \frac{1}{2^{k}} \sum_{m=0}^{2^{k} N_{0}-1} g\left(\frac{m}{2^{k}}\right),}_{E}
$$

where $g(t)=\nabla f(\mathbf{r}(t)) \cdot \mathbf{n}(\mathbf{r}(t))$ is Riemann-integrable on $\left[0, N_{0}\right]$ because $f, r_{1}, r_{2} \in \mathcal{C}^{1}$. We use the theorem of the Riemann sum with $a=0, b=N_{0}$, and $n=2^{k} N_{0}$ to obtain

$$
E=-\int_{0}^{N_{0}} \nabla f(\mathbf{r}(t)) \cdot \mathbf{n}(\mathbf{r}(t)) \mathrm{d} t
$$

Likewise, we use (4), (7), (8), and the theorem of the Riemann sum with $a=0, b=N_{0}, n=2^{k} N_{0}$, and $g(t)=g_{1}(\mathbf{r}(t)) \dot{r}_{2}(t)$, where $g$ is Riemann-integrable on $\left[0, N_{0}\right]$ because $f \in \mathcal{C}^{1}$, to obtain $\lim _{k \rightarrow \infty} E_{\text {region }}\left(f, \mathbf{p}_{(k)}\right)=F$, where $F$ is defined by

$$
\begin{aligned}
F & =\frac{1}{|\Sigma|}\left(2 \int_{0}^{N_{0}} g_{1}(\mathbf{r}(t)) \dot{r}_{2}(t) \mathrm{d} t-\int_{0}^{N_{0}} g_{1}\left(\mathbf{r}_{\lambda}(t)\right) \dot{r}_{\lambda, 2}(t) \mathrm{d} t\right) \\
& =\frac{1}{|\Sigma|}\left(2 \oint_{C} g_{1}(\mathbf{r}) \mathrm{d} r_{2}-\oint_{C_{\lambda}} g_{1}\left(\mathbf{r}_{\lambda}\right) \mathrm{d} r_{\lambda, 2}\right),
\end{aligned}
$$

where $C$ and $C_{\lambda}$ are the positive oriented contours that describe $\mathbf{r}$ and $\mathbf{r}_{\lambda}=\left(r_{\lambda, 1}, r_{\lambda, 2}\right)$, respectively. We use Green's theorem with $M=g_{1}\left(r_{1}, r_{2}\right)=\int_{-\infty}^{r_{1}} f\left(\tau, r_{2}\right) \mathrm{d} \tau$ and $Q=0$. We obtain

$$
\begin{aligned}
F & =\frac{1}{|\Sigma|}\left(2 \iint_{\Omega} f(\mathbf{r}) \mathrm{d} r_{1} \mathrm{~d} r_{2}-\iint_{\Omega_{\lambda}} f\left(\mathbf{r}_{\lambda}\right) \mathrm{d} r_{\lambda_{1}} \mathrm{~d} r_{\lambda_{2}}\right) \\
& =\frac{1}{|\Sigma|}\left(\iint_{\Omega} f(\mathbf{r}) \mathrm{d} r_{1} \mathrm{~d} r_{2}-\iint_{\Omega_{\lambda} \backslash \Omega} f(\mathbf{r}) \mathrm{d} r_{1} \mathrm{~d} r_{2}\right) .
\end{aligned}
$$

For $\Sigma\left(\mathbf{p}_{(k)}\right)$, we apply the same reasoning as previously, using first the theorem of the Riemann sum and then Green's theorem, to obtain

$$
\Sigma\left(\mathbf{p}_{(k)}\right) \underset{k \rightarrow \infty}{\longrightarrow} \Sigma=\iint_{\Omega} \mathrm{d} r_{1} \mathrm{~d} r_{2} .
$$

\section{REFERENCES}

[1] M. Kass, A. Witkin, and D. Terzopoulos, "Snakes: Active contour models," Int. J. Comput. Vis., vol. 1, no. 4, pp. 321-331, Jan. 1987.

[2] A. Blake and M. Isard, Active Contours: The Application of Techniques From Graphics, Vision, Control Theory and Statistics to Visual Tracking of Shapes in Motion, 1st ed. New York, NY, USA: Springer-Verlag, 1998.

[3] X. Bresson, S. Esedoglu, P. Vandergheynst, J.-P. Thiran, and S. Osher, "Fast global minimization of the active contour/snake model," J. Math. Imag. Vis., vol. 28, no. 2, pp. 151-167, 2007.

[4] C. Zimmer and J. C. Olivo-Marin, "Coupled parametric active contours," IEEE Trans. Pattern Anal. Mach. Intell., vol. 27, no. 11, pp. 1838-1842, Nov. 2005.

[5] A. Dufour, R. Thibeaux, E. Labruyere, N. Guillen, and J.-C. Olivo-Marin, "3-D active meshes: Fast discrete deformable models for cell tracking in 3-D time-lapse microscopy," IEEE Trans. Image Process., vol. 20, no. 7, pp. 1925-1937, Jul. 2011.

[6] J. Tang and S. T. Acton, "Vessel boundary tracking for intravital microscopy via multiscale gradient vector flow snakes," IEEE Trans. Biomed. Eng., vol. 51, no. 2, pp. 316-324, Feb. 2004.

[7] R. Delgado-Gonzalo, V. Uhlmann, D. Schmitter, and M. Unser, "Snakes on a plane: A perfect snap for bioimage analysis," IEEE Signal Process. Mag., vol. 32, no. 1, pp. 41-48, Jan. 2015.

[8] B. Li and S. T. Acton, "Active contour external force using vector field convolution for image segmentation," IEEE Trans. Image Process., vol. 16, no. 8, pp. 2096-2106, Aug. 2007. 
[9] J. Cardinale, G. Paul, and I. F. Sbalzarini, "Discrete region competition for unknown numbers of connected regions," IEEE Trans. Image Process., vol. 21, no. 8, pp. 3531-3545, Aug. 2012.

[10] N. Dyn and E. Farkhi, "Spline subdivision schemes for compact sets. A survey," Serdica Math. J., vol. 28, no. 4, pp. 349-360, 2002.

[11] C. Conti and L. Romani, "Algebraic conditions on non-stationary subdivision symbols for exponential polynomial reproduction," J. Comput. Appl. Math., vol. 236, no. 4, pp. 543-556, Sep. 2011.

[12] M. Charina, C. Conti, and L. Romani, "Reproduction of exponential polynomials by multivariate non-stationary subdivision schemes with a general dilation matrix," Numerische Math., vol. 127, no. 2, pp. 223-254, Jun. 2014.

[13] P. Novara and L. Romani, "Building blocks for designing arbitrarily smooth subdivision schemes with conic precision," J. Comput. Appl. Math., vol. 279, pp. 67-79, May 2015.

[14] I. Daubechies, Ten Lectures on Wavelets. Philadelphia, PA, USA: SIAM, 1992.

[15] F. Orderud and S. I. Rabben, "Real-time 3D segmentation of the left ventricle using deformable subdivision surfaces," in Proc. Comput. Vis. Pattern Recognit., 2008, pp. 1-8.

[16] J. Hug, C. Brechbühler, and G. Székely, "Tamed snake: A particle system for robust semi-automatic segmentation," in Proc. Int. Conf. Med. Image Comput. Comput.-Assist. Intervent., vol. 1679. 1999, pp. 106-115.

[17] D. Doo and M. Sabin, "Behaviour of recursive division surfaces near extraordinary points," Comput.-Aided Design, vol. 10, no. 6, pp. 356-360, 1978.

[18] N. Dyn, D. Levin, and J. A. Gregory, "A 4-point interpolatory subdivision scheme for curve design," Comput. Aided Geometric Design, vol. 4, no. 4, pp. 257-268, 1987.

[19] N. Dyn, D. Levin, and A. Luzzatto, "Exponentials reproducing subdivision schemes," Found. Comput. Math., vol. 3, no. 2, pp. 187-206, May 2003.

[20] C. V. Beccari, G. Casciola, and L. Romani, "Construction and characterization of non-uniform local interpolating polynomial splines," J. Comput. Appl. Math., vol. 240, pp. 5-19, Mar. 2013.

[21] M. Antonelli, C. V. Beccari, and G. Casciola, "A general framework for the construction of piecewise-polynomial local interpolants of minimum degree," Adv. Comput. Math., vol. 40, no. 4, pp. 945-976, Aug. 2014.

[22] J. Warren and H. Weimer, Subdivision Methods for Geometric Design: A Constructive Approach, 1st ed. San Francisco, CA, USA: Morgan Kaufmann, 2001.

[23] N. Dyn, "Subdivision schemes in CAGD," Adv. Numer. Anal., vol. 2, pp. 36-104, 1992.

[24] C. Conti and K. Hormann, "Polynomial reproduction for univariate subdivision schemes of any arity," J. Approx. Theory, vol. 163, no. 4, pp. 413-437, Apr. 2011.

[25] N. Dyn, J. A. Gregory, and D. Levin, "Analysis of uniform binary subdivision schemes for curve design," Constructive Approx., vol. 7, no. 1, pp. 127-147, Dec. 1991.

[26] N. Dyn, D. Levin, and J. Yoon, "Analysis of univariate nonstationary subdivision schemes with application to Gaussian-based interpolatory schemes," SIAM J. Math. Anal., vol. 39, no. 2, pp. 470-488, 2007.

[27] O. Rioul, "Simple regularity criteria for subdivision schemes," SIAM J. Math. Anal., vol. 23, no. 6, pp. 1544-1576, 1992.

[28] L. H. Staib and J. S. Duncan, "Boundary finding with parametrically deformable models," IEEE Trans. Pattern Anal. Mach. Intell., vol. 14 no. 11, pp. 1061-1075, Nov. 1992.

[29] P. Brigger, J. Hoeg, and M. Unser, "B-spline snakes: A flexible tool for parametric contour detection," IEEE Trans. Signal Process., vol. 9, no. 9, pp. 1484-1496, Sep. 2000.

[30] M. Jacob, T. Blu, and M. Unser, "A unifying approach and interface for spline-based snakes," in Proc. Int. Symp. Med. Imag., Imag. Process. (MI), San Diego, CA, USA, Feb. 2001, pp. 340-347.

[31] M. A. T. Figueiredo, J. M. N. Leito, and A. K. Jain, "Unsupervised contour representation and estimation using B-splines and a minimum description length criterion," IEEE Trans. Image Process., vol. 9, no. 6, pp. 1075-1087, Jun. 2000.

[32] M. Jacob, T. Blu, and M. Unser, "Efficient energies and algorithms for parametric snakes," IEEE Trans. Image Process., vol. 13, no. 9, pp. 1231-1244, Sep. 2004.

[33] P. Thévenaz, R. Delgado-Gonzalo, and M. Unser, "The ovuscule," IEEE Trans. Pattern Anal. Mach. Intell., vol. 33, no. 2, pp. 382-393, Feb. 2011.

[34] N. Ray, B. Chanda, and J. Das, "A fast and flexible multiresolution snake with a definite termination criterion," Pattern Recognit., vol. 34, no. 7, pp. 1483-1490, 2001
[35] P. Brigger and M. Unser, "Multi-scale B-spline snakes for general contour detection," in Proc. Conf. Math. Imag., Wavelet Appl. Signal Image Process., vol. 3458. Jul. 1998, pp. 92-102.

[36] B. Leroy, I. L. Herlin, and L. D. Cohen, "Multi-resolution algorithms for active contour models," in Proc. ICAOS, 1996, pp. 58-65.

[37] S. Dubuc and G. Deslauriers, "Symmetric iterative interpolation processes," Constructive Approx., vol. 5, no. 1, pp. 49-68, 1989.

[38] C. Conti, L. Gemignani, and L. Romani, "From symmetric subdivision masks of Hurwitz type to interpolatory subdivision masks," Linear Algebra Appl., vol. 431, no. 10, pp. 1971-1987, 2009.

[39] N. Saito and G. Beylkin, "Multiresolution representations using the autocorrelation functions of compactly supported wavelets," IEEE Trans. Signal Process., vol. 41, no. 12, pp. 3584-3590, Dec. 1993.

[40] I. Daubechies, "Orthonormal bases of compactly supported wavelets," Commun. Pure Appl. Math., vol. 41, no. 7, pp. 909-996, 1988. [Online]. Available: http://dx.doi.org/10.1002/cpa.3160410705

[41] C. Vonesch, T. Blu, and M. Unser, "Generalized Daubechies wavelet families," IEEE Trans. Signal Process., vol. 55, no. 9, pp. 4415-4429, Sep. 2007.

[42] J. Lane and R. Riesenfeld, "A theoretical development for the computer generation and display of piecewise polynomial surfaces," IEEE Trans. Pattern Anal. Mach. Intell., vol. 2, no. 1, pp. 35-46, Jan. 1980.

[43] L. Romani, "From approximating subdivision schemes for exponential splines to high-performance interpolating algorithms," J. Comput. Appl. Math., vol. 224, no. 1, pp. 383-396, Feb. 2009.

[44] C. Conti, L. Gemignani, and L. Romani, "From approximating to interpolatory non-stationary subdivision schemes with the same generation properties," Adv. Comput. Math., vol. 35, nos. 2-4, pp. 217-241, 2011.

[45] M. Unser and T. Blu, "Cardinal exponential splines: Part I-Theory and filtering algorithms," IEEE Trans. Signal Process., vol. 53, no. 4 , pp. 1425-1438, Apr. 2005.

[46] W. H. Press, S. A. Teukolsky, W. T. Vetterling, and B. P. Flannery, Numerical Recipes: The Art of Scientific Computing, 3rd ed. Cambridge, U.K.: Cambridge Univ. Press, 1986.

[47] P. G. Crichton, C. Affourtit, and A. L. Moore, "Identification of a mitochondrial alcohol dehydrogenase in Schizosaccharomyces pombe: New insights into energy metabolism," Biochem. J., vol. 401, no. 2, pp. 459-464, Jan. 2007.

[48] G. Serjeant and B. Serjeant, Sickle Cell Disease, 3rd ed. London, U.K.: Oxford Univ. Press, 2001.

[49] A. Badoual, D. Schmitter, and M. Unser, "Locally refinable parametric snakes," in Proc. IEEE Int. Conf. Image Process. (ICIP), Quebec City, QC, Canada, Sep. 2015, pp. 354-358, paper TEC-P21.2.

[50] F. de Chaumont et al., "Icy: An open bioimage informatics platform for extended reproducible research," Nature Methods, vol. 9, no. 7, pp. 690-696, Jul. 2012.

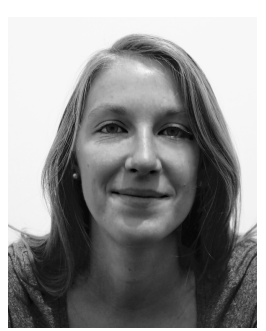

Anaiis Badoual received the M.Sc. degree in information technology and communication for health from Télécom Physique Strasbourg, France, and the M.Sc. degree in image processing from the University of Strasbourg, in 2014. She is currently pursuing the Ph.D. degree with the Biomedical Imaging Group, École Polytechnique Fédérale de Lausanne, Switzerland, under the direction of M. Unser. Her research interests include subdivision theory, deep learning, and segmentation problems.

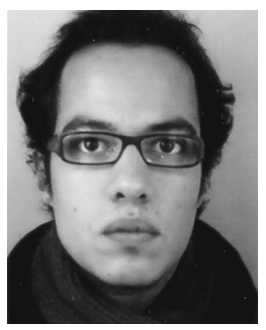

Daniel Schmitter received the master's degree in bioengineering and biomedical technologies from the École Polytechnique Fédérale de Lausanne (EPFL), Switzerland, in 2013, where he is currently pursuing the Ph.D. degree with the Biomedical Imaging Group. He is involved in spline-based shape representation and segmentation problems. He was with the Advanced Clinical Imaging Technology Group, Siemens, at the Center for Biomedical Imaging, Switzerland, where he was one of the main contributors involved in brain-imaging software and related image-processing algorithms. He has developed several segmentation and tracking software in the field of biomedical imaging. 


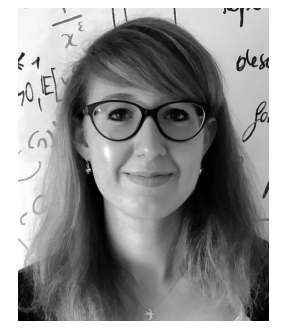

Virginie Uhlmann received the M.Sc. degree in bioengineering from the École Polytechnique Fédérale de Lausanne (EPFL), Switzerland, in 2012, where she is currently pursuing the Ph.D. degree with the Biomedical Imaging Group, under the direction of M. Unser. She completed her master's thesis in the Imaging Platform with the Broad Institute, Cambridge, MA, USA, under the supervision of A. Carpenter. She is involved in applied problem related to image segmentation and tracking, and on approximation and spline theory. Her research interests include image processing, computer vision, machine learning, and life sciences.

She received the competitive Excellence Fellowship at the master's level from EPFL, in 2011 and 2012, and was awarded the Best Student Paper Award from the 2014 IEEE International Conference on Image Processing (ICIP'14). She also received a Best Student Paper Award nomination at the IEEE International Symposium on Biomedical Imaging in 2015.

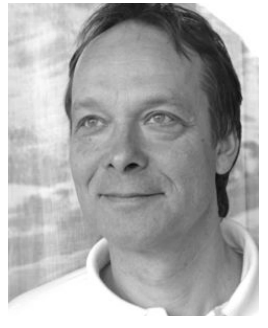

Michael Unser (M'89-SM'94-F'99) was with the Biomedical Engineering and Instrumentation Program, National Institutes of Health, Bethesda, USA, conducting research on bioimaging, from 1985 to 1997 . He is currently a Professor and Director of the Biomedical Imaging Group with the École Polytechnique Fédérale de Lausanne, Switzerland. He has published over 250 journal papers in his research fields. He is the author with P. Tafti of the book titled An Introduction to Sparse Stochastic Processes (Cambridge University Press, 2014). His primary area of investigation is biomedical image processing. $\mathrm{He}$ is internationally recognized for his research contributions to sampling theory, wavelets, the use of splines for image processing, stochastic processes, and computational bioimaging.

Prof. Unser is an EURASIP Fellow (2009), and a member of the Swiss Academy of Engineering Sciences. He is the recipient of several international prizes, including three IEEE-SPS Best Paper Awards and two Technical Achievement Awards from the IEEE (2008 SPS and EMBS 2010). He was an Associate Editor-in-Chief (2003-2005) of the IEEE TRANSACTIONS ON MEdical IMAGing. He is currently a member of the editorial boards of the SIAM J. Imaging Sciences, the IEEE J. Selected Topics in Signal Processing, and the Foundations and Trends in Signal Processing. He is the Founding Chair of the Technical Committee on Bio Imaging and Signal Processing (BISP) of the IEEE Signal Processing Society. 